\title{
Disminución del filtrado glomerular con la edad, un fenómeno
} normal

\author{
Decreased glomerular filtration with age, a normal phenomenon
}

Diego H. Ramírez-Leyva*

Instituto Mexicano del Seguro Social, Unidad de Medicina Familiar 1, Sonora, México

Leí con interés "Insuficiencia renal oculta y factores asociados en pacientes con enfermedades crónicas", de Balderas Vargas et al., ${ }^{1}$ publicado en el número 1 de 2020 de Gaceta Médica de México. En el artículo, los autores mencionan la edad como factor de riesgo para insuficiencia renal oculta, resultado que debe considerarse con reserva ya que el envejecimiento renal produce ese resultado sin que exista lesión.

Es importante explicar por qué los autores sugieren los 60 años como punto de corte para considerar la edad como factor de riesgo, ya que es normal que los individuos tengan función renal disminuida $(0.8 \mathrm{~mL} /$ hora por año) a partir de los 40 años o, incluso, antes. ${ }^{2}$ En la discusión del artículo no se menciona la explicación de este factor significativo. Desde mi opinión no aplica como factor de riesgo y solo corresponde al proceso normal de envejecimiento. Me parece más adecuado medir la función renal esperada según la edad y, posteriormente, valorar la presencia de insuficiencia renal oculta.,

En el artículo también se menciona el control de la diabetes mellitus y la hipertensión arterial como factor asociado, pero no se indica cómo fue establecido, lo cual afecta los resultados, ya que precisamente el control de las enfermedades crónicas es un factor determinante para la función renal..$^{5}$ En ese aspecto, la medición de la glucosa, hemoglobina glucosilada y presión arterial en su forma natural permitiría una mejor apreciación que la dicotomía control-descontrol. En ese sentido, el tiempo de evolución no se menciona en los resultados ni en la discusión. Desde mi perspectiva, esa variable modificaría los resultados.

\section{Bibliografía}

1. Balderas-Vargas NA, Legorreta-Soberanis J, Paredes-Solís S, Flores-Moreno M, Serrano-de los Santos FR, Andersson N. Insuficiencia renal oculta y factores asociados en pacientes con enfermedades crónicas. Gac Med Mex. 2020;156:11-16.

2. Davies DF, Shock NW. Age changes in glomerular filtration rate, effective renal plasma flow, and tubular excretory capacity in adult males. $J$ Clin Invest. 1950:29:496-507.

3. Otero-González A. Envejecimiento y función renal. Mecanismos de predicción y progresión. Nefrol Sup Ext. 2011;2:119-130.

4. Muntner P. Longitudinal measurements of renal function. Semin Nephrol. 2009;29:650-657.

5. Maclsaac RJ, Jerums G, Ekinci El. Effects of glycaemic management on diabetic kidney disease. World J Diabetes. 2017;8:172-186.

\section{Correspondencia:}

*Diego H. Ramírez-Leyva

E-mail: diegooram@hotmail.com
Gac Med Mex. 2020;156:359

Disponible en PubMed

www.gacetamedicademexico.com

CC BY-NC-ND (http://creativecommons.org/licenses/by-nc-nd/4.0/). 